\title{
A 28-year-old military veteran with nightmares and insomnia
}

\author{
Allison Crawford MD MA
}

Competing interests: None declared.

This article has been peer reviewed.

\section{Correspondence to:}

Allison Crawford,

allison.crawford@utoronto.ca

CMAJ 2014. DOI:10.1503

/cmaj.130187
A 28-year-old military veteran, a married father of two, presents to his primary care physician, reporting problems with insomnia and nightmares.

\section{Should this patient be assessed for post- traumatic stress disorder?}

Given the patient's military background and the symptoms of insomnia and nightmares, which are consistent with posttraumatic stress disorder (PTSD), this diagnosis should be considered.

Further inquiry indicates that this patient returned from deployment in Afghanistan two years earlier. Previous exposure to potentially traumatic events in the military (e.g., combat, witnessing injury or death of comrades, witnessing atrocity) should prompt screening for the presence of this disorder. ${ }^{1-3}$ Among civilians, exposure to events that may have resulted in fear of death or bodily injury, or witnessing death or bodily injury, also indicate a need to screen for psychological distress or trauma. ${ }^{1-3}$ Many patients will not spontaneously offer this information. The Primary Care PTSD screen (Box 1) is a brief, validated instrument (sensitivity 0.78 , specificity 0.87 ) for use in primary care settings, including with combat veterans. ${ }^{1}$

There is no clear evidence of an elevated risk

\section{Box 1: Screening for posttraumatic stress disorder: the Primary Care} PTSD Screen ${ }^{1}$

In your life, have you ever had any experience that was so frightening, horrible or upsetting that, in the past month,

- you have had nightmares about it or thought about it when you did not want to? Yes/No

- you tried hard not to think about it or went out of your way to avoid situations that reminded you of it? Yes/No

- you were constantly on guard, watchful or easily startled? Yes/No

- you felt numb or detached from others, activities or your surroundings? Yes/No

The screening result should be considered "positive" if a patient answers "yes" to any three items. of mental health problems related to military deployment per se. However, there is evidence of a strong link between exposure to combat and/or the witnessing of atrocities and the development of mental health disorders, including PTSD. A dose-response relationship has been shown, with greater exposure to combat or atrocity more likely to lead to PTSD. ${ }^{2}$

\section{Does this patient have PTSD?}

Exposure to a traumatic event is required for a diagnosis of PTSD, but not all exposure to potentially traumatic events leads to development of the disorder. According to the Diagnostic and Statistical Manual of Mental Disorders, fifth edition (DSM-V), the diagnosis of PTSD requires the presence of symptoms, for more than one month, in each of four categories: intrusion symptoms (e.g., intrusive, distressing memories or nightmares of the traumatic event or events), avoidance (e.g., persistent effortful avoidance of thoughts, feelings, people or places that act as reminders of the traumatic events), negative alterations in cognition and mood (e.g., persistent and/or distorted negative expectations about oneself, others or the world) and alterations in arousal and reactivity (e.g., irritable or aggressive behaviour, sleep disturbance). In PTSD, these symptoms result in clinically significant distress or impairment of functioning. Symptoms lasting for less than one month are suggestive of an acute stress disorder (also as outlined in DSM-V).

Comorbidity, which can worsen the prognosis, occurs in $79 \%-88 \%$ of individuals with PTSD. ${ }^{4}$ As such, this patient should also be screened for depression, anxiety and substance abuse..$^{3,4}$

Is this patient at increased risk of suicide? A recent review of the relation between PTSD and suicide risk did not show an elevated risk of suicide completion by those with PTSD. However, an association was found between PTSD 
and prior suicide attempts, as well as current suicidal ideation; the risk was greater in the presence of concurrent depression. ${ }^{5}$ This patient should be asked about current suicidal thoughts or plans and should also be questioned about access to weapons or other lethal means.

\section{What are some initial interventions that can be used?}

Box 2 offers a general approach to first-line treatment of PTSD in primary care. ${ }^{3,4,6}$ Psychological interventions specifically developed for PTSD have the strongest evidence for efficacy, particularly trauma-focused cognitive behavioural therapies that address maladaptive thinking (e.g., responsibility, guilt related to the traumatic incident) and exposure therapies that involve imaginal reliving of aspects of the traumatic events. ${ }^{3,4}$ These interventions are significantly more effective than supportive treatment and nonspecific therapies. Notably, for nonsymptomatic individuals, the use of psychological intervention after exposure to trauma is potentially harmful. ${ }^{3}$

The first-line pharmacologic treatment for PTSD is selective serotonin reuptake inhibitors. ${ }^{3,4,6}$ Because partial response to treatment is common, approaches that combine psychotherapy and pharmacotherapy are frequently used. ${ }^{4}$ Pharmacologic therapy may be considered as the initial intervention if the patient's condition is not sufficiently stable to allow him or her to engage in trauma-focused therapy, if the patient is unwilling to engage in therapy or if there is a high level of dissociative symptoms. ${ }^{3}$ There is modest evidence for augmentation of therapy with an atypical antipsychotic (i.e., risperidone or olanzapine) in patients with no response to monotherapy with selective serotonin reuptake inhibitors or serotonin-norepinephrine reuptake inhibitors. ${ }^{3,6}$ However, the potential adverse effect of atypical antipsychotic agents on the patient's metabolic profile should be taken into consideration. There is no evidence to support the use of benzodiazepines, which may cause harm.,

\section{When should referral be considered?}

Primary care physicians may decide to involve intraprofessional team members, particularly if they do not have expertise in treating psychological trauma, in working with military personnel or in delivering evidence-based, trauma-focused psychotherapy. Referral to a psychiatrist should be considered if acute safety concerns are identified or if the patient's PTSD symptoms are refractory to the treatments outlined above. Specialized care is often available for military personnel and their families (Box 3).

\section{What is the long-term prognosis for this patient?}

Trauma-focused therapy has been shown to significantly reduce symptoms of PTSD, to decrease symptom severity and to lessen symptoms of depression and anxiety. ${ }^{3}$ To date, no adequate trials have compared responses to traumafocused psychotherapy and pharmacologic treat-

\section{Box 2: Stepped approach to primary care intervention for PTSD ${ }^{3,4,6}$}

Step 1

- Establish rapport and trust

- Perform physical examination and laboratory investigations as guided by initial general assessment

- Screen for PTSD (using Primary Care PTSD screen; see Box 1) and associated comorbidities

- Assess immediate safety

- Engage in watchful waiting if $<2$ wk since exposure to trauma

- Offer PTSD psychoeducation (e.g., to normalize response, instil hope)

Step 2

- Offer or refer for evidence-based psychotherapy (e.g., trauma-focused cognitive behavioural therapy)

- Avoid benzodiazepines, hypnotics and opiates

- Provide resources to patient and family

Step 3

- If there is only partial response to psychotherapy or the patient declines psychotherapy, consider treatment with an SSRI

- If patient presents with severe symptoms of PTSD, consider a combination of SSRI and psychotherapy

- Monitor for suicidal ideation

Step 4

- For patients with a response to SSRI therapy, continue treatment for $1 \mathrm{yr}$

- If there is no response, change or augment medication (i.e., risperidone or olanzapine)

- Refer to a psychiatry or mental health professional with expertise in PTSD and/or military medicine

- Reconsider comorbidity

Note: PTSD = posttraumatic stress disorder, SSRI = selective serotonin reuptake inhibitor.

\section{Box 3: Resources on posttraumatic stress disorder}

- General information: International Society for Traumatic Stress Studies: www.ISTSS.org

- Canadian Forces Health Services: www.forces.gc.ca/en/caf-community-health -services/index.page

- Operational Stress Injury Social Support: www.osiss.ca/

- US Department of Veterans Affairs, National Center for PTSD: www.ptsd.va.gov

- Clinical practice guidelines:

- Australian Centre for Posttraumatic Mental Health: www.nhmrc.gov.au I_files_nhmrdpublications/attachments/mh15.pdf

- Canadian Psychiatric Association: http://publications.cpa-apc.org /browse/documents/213

- International Society for Traumatic Stress Studies: www.istss.org /TreatmentGuidelines/5168.htm 
ment. ${ }^{3}$ However, among people with a partial response to pharmacologic agents, further improvement has been documented with psychotherapy. ${ }^{6}$ Typically, military personnel experience a more chronic course of PTSD and their response to interventions is less robust ${ }^{3}$ than people with PTSD from nonmilitary causes; however, current evidence suggests that this may not be true for veterans of more recent wars, ${ }^{4,6}$ such as this patient.

\section{The case revisited}

Initial psychoeducation allowed the patient to understand his nightmares and sleep difficulties within the context of PTSD. He and his family sought resources and support through a military support program. His primary care physician referred him for trauma-focused cognitive behavioural therapy, which resulted in partial reduction in his symptoms. After 10 weeks of treatment, he was also started on paroxetine, in combination with the cognitive behavioural therapy. He responded well to the medication after six weeks and will continue taking it for one year.

\section{References}

1. Prins A, Oulmette P, Kimerling R, et al. The primary care PTSD screen (PC-PTSD): development and operating characteristics. Prim Care Psychiatry 2003;9:9-14.

2. Sareen J, Cox BJ, Afifi TO, et al. Combat and peacekeeping operations in relation to prevalence of mental disorders and perceived need for mental health care. Findings from a large representative sample of military personnel. Arch Gen Psychiatry 2007;64:843-52.

3. Australian guidelines for the treatment of adults with acute stress disorder and posttraumatic stress disorder. Melbourne (AU): National Health and Medical Research Council; 2007.

4. Pratchett LC, Daly K, Bierer LM, et al. New approaches to combining pharmacotherapy and psychotherapy for posttraumatic stress disorder. Expert Opin Pharmacother 2011;12:2339-54.

5. Krysinska K, Lester D. Post-traumatic stress disorder and suicide risk: a systematic review. Arch Suicide Res 2010;14:1-23.

6. Ipser JC, Stein DJ. Evidence-based pharmacotherapy of posttraumatic stress disorder (PTSD). Int J Neuropsychopharmacol $2012 ; 15: 825-40$

Affiliations: Northern Psychiatric Outreach Program and Telepsychiatry, Centre for Addiction and Mental Health and Department of Psychiatry, University of Toronto, Toronto, Ont.

\section{How you can get involved in the CMA!}

The CMA is committed to providing leadership for physicians and promoting the highest standard of health and health care for Canadians. To strengthen the association and be truly representative of all Canadian physicians the CMA needs to hear from members interested in serving in elected positions and on appointed committees and advisory groups. The CMA structure comprises both governing bodies and advisory bodies either elected by General Council or appointed by the CMA Board of Directors. The Board of Directors - elected by General Council - has provincial/territorial, resident and student representation, is responsible for the overall operation of the CMA and reports to General Council on issues of governance.

CMA committees advise the Board of Directors and make recommendations on specific issues of concern to physicians and the public. Five core committees mainly consist of regional, resident and student representation while other statutory and special committees and task forces consist of individuals with interest and expertise in subject-specific fields. Positions on one or more of these committees may become available in the coming year.

For further information on how you can get involved please go to http://www.cma.ca/membercentre/how-you-can-get-involved, or contact

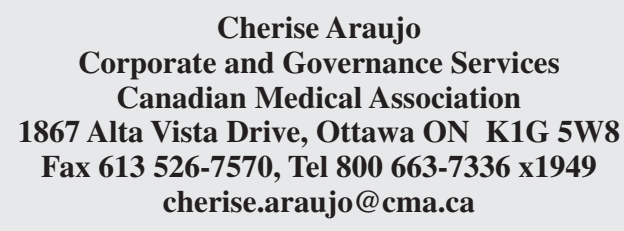

By getting involved, you will have an opportunity to make a difference.

We hope to hear from you!

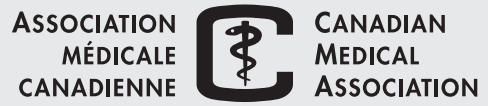

